\title{
Pilot study of hepatic arterial infusion chemotherapy with gemcitabine and 5-fluorouracil for patients with postoperative liver metastases from pancreatic cancer
}

\author{
HIDEHIRO TAJIMA $^{1}$, TETSUO OHTA ${ }^{1}$, HIROHISA KITAGAWA ${ }^{1}$, SEISHO SAKAI ${ }^{1}$, ISAMU MAKINO ${ }^{1}$, \\ HIRONORI HAYASHI ${ }^{1}$, KATSUNOBU OYAMA ${ }^{1}$, HISATOSHI NAKAGAWARA ${ }^{1}$, HIDETO FUJTTA ${ }^{1}$, \\ ICHIRO ONISHI $^{1}$, HIROYUKI TAKAMURA $^{1}$, ITASU NINOMIYA $^{1}$, SACHIO FUSHIDA $^{1}$, \\ TAKASHI TANI ${ }^{1}$, TAKASHI FUJIMURA ${ }^{1}$, WATARU KODA ${ }^{2}$, TETSUYA MINAMI $^{2}$, \\ YASUJI RYU ${ }^{2}$, JUNICHIRO SANADA ${ }^{2}$, TOSHIFUMI GABATA ${ }^{2}$ and OSAMU MATSUI ${ }^{2}$ \\ Departments of ${ }^{1}$ Gastroenterologic Surgery, and ${ }^{2}$ Radiology, Division of Cancer Medicine, \\ Graduate School of Medicine Science, Kanazawa University, Kanazawa 920-8641, Japan
}

Received October 3, 2010; Accepted December 28, 2010

DOI: $10.3892 / \mathrm{etm} .2011 .190$

\begin{abstract}
Hepatic metastasis is a common cause of treatment failure after curative resection of pancreatic cancer. We report a pilot study of hepatic arterial infusion (HAI) chemotherapy with gemcitabine and 5-fluorouracil (5-FU) for postoperative liver metastases from pancreatic cancer. Five patients who had undergone curative resection of liver metastases from pancreatic cancer received HAI of gemcitabine and 5-FU between October 2008 and September 2010 at Kanazawa University Hospital. Gemcitabine at a dose of $800 \mathrm{mg}$ was infused over $30 \mathrm{~min}$ via a bedside pump. After gemcitabine administration, $250 \mathrm{mg}$ of 5 -FU was infused continuously over $24 \mathrm{~h}$ on days $1-5$, comprising one cycle of therapy. These treatment cycles were continued biweekly. In the evaluation according to RECIST criteria, a partial response was obtained in 2 of the 5 cases, with stable disease being achieved in the remaining 3 cases (response rate, 100\%). In 4 of the 5 cases, a decrease in serum tumor marker CA19-9 was observed after $10 \mathrm{HAI}$ treatment cycles. The median time to treatment failure was 10 months (range 3-17). As to adverse events, leukocytopenia was grade 3 in 1 of 4 affected cases and all 5 were anemic, although 4 of the 5 cases had anemia prior to HAI therapy. Grade 2 thrombocytopenia was observed in 2 cases. No nonhematologic events, such as nausea, diarrhea, liver injury and neuropathy, occurred. There were no life-threatening toxicities, but 4 cases $(80 \%)$ developed catheter complications, and the HAI catheter and subcutaneous implantable port system
\end{abstract}

Correspondence to: Dr Hidehiro Tajima, Department of Gastroenterologic Surgery, Graduate School of Medicine Science, Kanazawa University, 13-1 Takaramachi, Kanazawa 920-8641, Japan E-mail: hidetaji@staff.kanazawa-u.ac.jp

Key words: pancreatic cancer, liver metastasis, hepatic arterial infusion, chemotherapy, gemcitabine, 5-fluorouracil had to be removed. HAI delivers high doses of chemotherapeutic agents directly into tumor vessels, producing increased regional levels with greater efficacy and a lower incidence/ severity of systemic side effects. In conclusion, HAI chemotherapy is useful and safe for the treatment of malignancies confined to the liver.

\section{Introduction}

Pancreatic cancer is one of the major causes of cancer-related death globally, with a 5-year survival rate of less than 5\% $(1,2)$. For patients with localized disease, radical surgery may provide long-term benefits. However, even in patients who undergo resection, the reported 5-year survival rate remains in the range of $7-24 \%$, and median survival is only approximately 1 year in most series, indicating that surgery alone is generally inadequate. Even after curative resection, patients with pancreatic cancer face a $50-80 \%$ local recurrence rate and a $25-50 \%$ chance of developing distant metastases (3).

Gemcitabine, a deoxycytidine analogue that competes for incorporation into DNA thereby inhibiting its synthesis, is the key drug employed in the treatment of pancreatic cancer. Adjuvant chemotherapy with gemcitabine improves, although to a limited degree, the survival of patients with resectable pancreatic adenocarcinoma as compared to resection alone (4). However, a major drawback of adjuvant therapy for pancreatic cancer is that $20-30 \%$ of patients cannot receive the designated therapy as a result of postoperative complications, delayed surgical recovery and/or early disease recurrence $(5,6)$.

Hepatic arterial infusion (HAI) of chemotherapeutic agents is a treatment option in patients with primary or metastatic hepatic malignancies that are confined to the liver. The use of HAI chemotherapy is soundly based on physiological and pharmacological factors. First, liver metastases that grow beyond 2-3 $\mathrm{mm}$ depend on the hepatic artery for vascularization, whereas normal liver tissues are perfused by the portal vein $(7,8)$. Second, HAI therapy allows for drug delivery to 
Table I. Patient characteristics.

\begin{tabular}{lccccc}
\hline Case no. & 1 & 2 & 3 & 4 & 5 \\
\hline Age & 61 & 62 & 69 & 71 & 60 \\
Gender & F & M & M & M & F \\
Performance status & 0 & 0 & 0 & 0 & 0 \\
Tumor location & H & H & BT & BT & BT \\
Stage at surgery & IV A & III & II & III & IV A \\
Resection & 0 & 0 & 0 & 0 & 0 \\
Preoperative chemotherapy & + & + & - & - & - \\
Postoperative chemotherapy & - & + & 3 & 10 & + \\
Interval between surgery & 5 & 10 & & & 5 \\
and liver metastasis (months) & & 4 & 1.84 & 1.51 & 1.07 \\
No. of liver metastases & 2 & 1.7 & 1.30 & 1.27 \\
Body surface area (m ${ }^{2}$ ) & 1.60 & 1.2 & & & 0.90 \\
Standard liver volume (liters) & 1.13 & & & & \\
\hline
\end{tabular}

hepatic metastases not achievable by systemic administration, particularly for drugs with a high systemic clearance rate (9). Third, first-pass hepatic extraction of certain drugs results in lower systemic concentrations and hence, few systemic toxicities (10). Phase I studies of HAI chemotherapy with gemcitabine in patients with liver malignancies have recently been reported (8-10).

We recently described a patient with pancreatic head cancer with postoperative liver metastases, judged to be an unsuitable candidate for systemic chemotherapy due to leukocytopenia, who was safely treated with gemcitabine plus 5-fluorouracil (5-FU) HAI (11). It was suggested that HAI chemotherapy is useful and safe for the treatment of postoperative metastatic tumors confined to the liver, even in patients in poor general condition. To improve the therapeutic results of resected pancreatic cancer, it is vital to optimize the postoperative management of liver metastases, which frequently constitute the major factor determining prognosis. We designed a pilot study for patients with postoperative liver metastases from pancreatic cancer in which an arterial infusion of gemcitabine and 5-FU was delivered directly to the liver, and report our experience herein.

\section{Patients and methods}

Patient eligibility. Five patients with postoperative liver metastases from pancreatic cancer underwent HAI with gemcitabine and 5-FU between October 2008 and September 2010 at Kanazawa University Hospital. The baseline characteristics of the patients are listed in Table I. The patients selected for this study had metastases confined to the liver after curative (R0) resection of the pancreatic primary adenocarcinoma. The male-to-female ratio was 3:2. The median patient age was 64.6 years (range 60-71). The Eastern Cooperative Oncology Group performance status was 0 in all patients in this study. Two patients had received preoperative chemotherapy with gemcitabine and oral S-1, and adjuvant chemotherapy with gemcitabine had been administered to 3 of the 5 patients before the appearance of liver metastases. The interval between surgery and the appearance of liver metastases was 6.6 months (range 3-10). The median standard liver volume [SLV $(\mathrm{ml})=$ 706.2 x body surface area (BSA) + 2.4] of our patients was 1.12 liters (range 9.0-1.30) (12).

Written informed consent was obtained from each patient prior to enrollment in the study, and the treatment was undertaken with the approval of the local medical ethics committee.

Catheter placement and treatment regimen. An intrahepatic arterial catheter was percutaneously implanted after hepatic arteriography via a right femoral puncture. The catheter tip was placed at the proper hepatic artery by a radiologist. The catheter was then connected to a subcutaneous implantable port system, located in the right lower abdominal area. Then, an $800 \mathrm{mg}$ dose of gemcitabine was dissolved in $50 \mathrm{ml}$ of saline for administration over a 30 -min period using a bedside pump. After gemcitabine infusion, a 250-mg dose of 5-FU dissolved in $50 \mathrm{ml}$ of saline was infused continuously over $24 \mathrm{~h}$ on days 1-5, comprising one cycle of therapy. In Case 1, only $400 \mathrm{mg}$ of gemcitabine was administered because of the presence of leukocytopenia (11). Each treatment cycle was continued biweekly from hospital days 1-6.

Assessment of response. Physical examination, complete blood counts, biochemical tests, chest and abdominal X-rays were obtained before the start of each cycle. Serum CA19-9 was measured monthly and changes in this tumor marker were determined as the serum CA19-9 level before and after 10 HAI cycles. Follow-up contrast-enhanced CT scans were conducted upon completion of each 5 cycles, or earlier in cases showing clinical deterioration. The response rate was evaluated in accordance with Response Evaluation Criteria in Solid Tumors (RECIST) criteria (13). A complete response (CR) was defined as the disappearance of all evidence of disease and normalization of tumor markers persisting for at least 2 weeks. A partial response (PR) was defined as a $>30 \%$ reduction on uni-dimensional tumor measurements, without 
Table II. Treatments and responses.

\begin{tabular}{|c|c|c|c|c|c|}
\hline Case no. & 1 & 2 & 3 & 4 & 5 \\
\hline GEM administration (cycles) & 13 & 40 & 23 & 10 & 8 \\
\hline 5-FU administration (cycles) & 10 & 10 & 10 & 10 & 6 \\
\hline Response & SD & PR & PR & PR & $\mathrm{SD}$ \\
\hline TTF (months) & 15 & 17 & 7 & 8 & 3 \\
\hline Another metastatic lesion & $\mathrm{L}, \mathrm{N}$ & $\mathrm{N}$ & $\operatorname{Lg}$ & $\mathrm{L}, \mathrm{Lg}$ & $\mathrm{N}$ \\
\hline Another chemotherapy & $\mathrm{Tx}$ & S-1 & S-1 & S-1 & S-1 \\
\hline Another therapy & $\mathrm{RT}$ & - & - & - & - \\
\hline Prognosis & $\mathrm{D}$ & A & A & A & A \\
\hline Survival after HAI (months) & 23 & 24 & 13 & 13 & 9 \\
\hline Catheter problems & + & + & - & + & + \\
\hline CA19-9 before HAI (U/ml) & 138 & 14 & 311 & 2,073 & 43,460 \\
\hline CA19-9 after 10 HAI cycles (U/ml) & 33 & 65 & 221 & 811 & 32,200 \\
\hline
\end{tabular}

GEM, gemcitabine; TTF, time to treatment failure; L, local recurrence; N, lymph node metastasis; Lg, lung metastasis; Tx, taxane; RT, radiation therapy; D, dead; A, alive.

the appearance of any new lesions or progression in any existing lesion. Progressive disease (PD) was defined as any of the following: i) a $20 \%$ increase in the sum of the products of all measurable lesions, ii) the appearance of any new lesion, or iii) the reappearance of any lesion that had previously disappeared. Stable disease (SD) was defined as a tumor response that did not fulfill the criteria for CR, PR or PD.

After 10 cycles, HAI of 5-FU was terminated and administration of oral S-1 was initiated. S-1 is an oral fluorinated pyrimidine developed by Taiho Pharmaceutical Co., Ltd. (Tokyo, Japan). Patients received gemcitabine HAI and administration of oral S-1 in the outpatient clinic for as long as possible, i.e., so long as they showed neither tumor re-growth nor the appearance of any new lesions, and were free of HAI catheter-related problems.

\section{Results}

The median follow-up duration was 15.6 months (range 8-23). In 4 cases, more than 10 cycles of HAI chemotherapy were administered. In a single case (Case 5), after the 8th cycle, the HAI catheter and subcutaneous implantable port system had to be removed because of a complication with the tube.

In the evaluation according to RECIST criteria, PR was obtained in 2 of the 5 cases, and SD was achieved in the other 3 cases (100\% response rate). Neither CR nor PD was observed. In 4 of the 5 cases, decreases in the serum tumor marker CA19-9 were observed after 10 cycles of HAI treatment.

The median time to treatment failure was 10 months (range 3-17). The initial disease progression factor was nodal metastasis in 3 cases and local recurrence plus lung metastasis in 2 cases (Table II). The overall median survival time was 22.4 months (range 9-24). In Case 1, the cause of death was progression of local recurrence, nodal metastases and re-growth of liver metastases after termination of HAI due to arterial thrombosis.

Adverse events are listed in Table III. Grade 3 leukocytopenia was observed in Case 1 , who could not receive
Table III. Treatment toxicities (NCI-CTC grade).

\begin{tabular}{llllll}
\hline Case no. & 1 & 2 & 3 & 4 & 5 \\
\hline Anemia & 2 & 2 & 1 & 1 & 2 \\
Leukocytopenia & $3^{\mathrm{a}}$ & 0 & 0 & 0 & 0 \\
Thrombocytopenia & 2 & 1 & 0 & 2 & 0 \\
Nausea & 0 & 0 & 0 & 0 & 0 \\
Diarrhea & 0 & 0 & 0 & 0 & 0 \\
Liver injury & 0 & 0 & 0 & 0 & 0 \\
Neuropathy & 0 & 0 & 0 & 0 & 0
\end{tabular}

${ }^{\text {aGrade }} 2$ before HAI was started.

adjuvant systemic chemotherapy because of grade 2 leukocytopenia prior to HAI. Leukocytopenia was not observed in any other cases. All cases were anemic, but 4 of the 5 had been anemic prior to HAI therapy. Grade 2 thrombocytopenia was observed in 2 cases. There were no non-hematologic events, such as nausea, diarrhea, liver injury (AST/ALT increase) and neuropathy. No life-threatening toxicities developed, but in 4 cases $(80 \%)$ catheter complications (arterial thrombosis or catheter dislocation) occurred, and the HAI catheter and subcutaneous implantable port system had to be removed.

\section{Discussion}

Pancreatic cancer is nearly always a fatal disease, with a 5 -year survival rate of less than 5\% $(1,2)$. Surgery remains the only curative option. Therefore, we usually perform radical pancreatic resection, including wide lymph node dissection and complete removal of the extra-pancreatic nerve plexus of the superior mesenteric artery or celiac axis, in patients with carcinoma of the pancreas in hopes of improving the prognosis $(14,15)$. Adjuvant chemotherapy improves, although 
to a limited degree, the survival of patients with resectable pancreatic adenocarcinoma as compared to resection alone (4). However, a major drawback of adjuvant therapy for pancreatic cancer is that $20-30 \%$ of patients cannot receive the designated therapy due to postoperative complications, delayed surgical recovery and/or early disease recurrence $(5,6)$. Theoretically, these drawbacks may be overcome by employing the neoadjuvant approach, so that more patients can receive potentially beneficial adjuvant treatment. Clinical studies of neoadjuvant chemo-(radio)-therapy in pancreatic cancer have recently been reported (16-22).

HAI chemotherapy, using fluorodeoxyuridine or 5-FU, has been studied most extensively in patients with liver metastases from colorectal cancer. Despite the significantly higher response rates to HAI than to intravenous infusion, most of these studies did not document a significant prolongation of survival, even in meta-analyses $(23,24)$. Arterial infusion chemotherapy with gemcitabine and 5-FU was recently reported for locally advanced pancreatic cancer and liver metastases from pancreatic cancer $(10,25,26)$. Moreover, in some phase I studies, HAI chemotherapy with gemcitabine was well tolerated up to $1,000 \mathrm{mg} / \mathrm{m}^{2}$ infused over $400 \mathrm{~min}(8,9)$.

According to the pharmacokinetics of gemcitabine, when $1,000 \mathrm{mg} / \mathrm{m}^{2}$ of gemcitabine is administered via intravenous infusion over $30 \mathrm{~min}$, the average maximum plasma concentrations reach $21,865 \pm 4,165 \mathrm{ng} / \mathrm{ml}$ by $15 \mathrm{~min}$. The flow volume of the proper hepatic artery is reportedly approximately $330 \mathrm{ml} / \mathrm{min}$ (27). When an 800-mg dose of gemcitabine is infused into the proper hepatic artery over a 30-min period, the local plasma concentration in the liver reaches approximately $80,000 \mathrm{ng} / \mathrm{ml}$ by $30 \mathrm{~min}$. Vogl et al reported that the maximum tolerated dose of HAI chemotherapy with gemcitabine was $1,400 \mathrm{mg} / \mathrm{m}^{2}$ (8). On the other hand, the plasma concentration of 5-FU with a $250 \mathrm{mg}$ infusion into the proper hepatic artery over a 24 -h period was 0.5 $\mu \mathrm{g} / \mathrm{ml}$. This concentration is equal to that obtained following administration of $30 \mathrm{mg} / \mathrm{kg}(1,350 \mathrm{mg}$ in the reported patient) of 5-FU over a 24-h period (28). Maruyama et al reported that when 1,000-1,500 $\mathrm{mg}$ of 5-FU was infused into the hepatic artery over a period of $5 \mathrm{~h}$, the maximum plasma concentration was $0.48 \mu \mathrm{g} / \mathrm{ml}$, on average, and no grade 3 adverse effects developed (29).

Super-selective HAI delivers high doses of chemotherapeutic agents into tumor vessels, producing increased regional levels with more effectiveness and a lower incidence/severity of systemic side effects. In this study, the response rate was $100 \%$, despite 4 of the 5 cases having received systemic chemotherapy with gemcitabine before HAI. Moreover, no severe toxicity developed with this therapy. Thus, HAI chemotherapy is safe and effective for postoperative liver metastasis from pancreatic carcinoma. The drawbacks of HAI chemotherapy include problems with the tube and the appearance of new lesions outside of the liver. In this study, 4 of 5 cases required removal of the HAI catheter and the subcutaneous implantable port system because of problems with the tube, and new lesions outside of the liver appeared in all 5 patients.

In a previous study, the amounts of chemotherapeutic agents to be delivered were determined based on BSA. However, SLV is more suitable than BSA for determining the drug amount to be administered in HAI chemotherapy. We advocate that in HAI the amounts of chemotherapeutic agents are determined based on SLV. A phase I study of HAI chemotherapy with gemcitabine and 5-FU is underway in patients with pancreatic cancer with postoperative metastases confined to be liver. In conclusion, HAI chemotherapy is useful and safe for the treatment of postoperative metastases from pancreatic cancer confined to the liver.

\section{References}

1. International Agency for Research on Cancer, World Health Organization. Globocan 2008. World Health Organization Web site. http://globocan.iarc.fr/. Accessed October 1, 2010.

2. Ishii H, Furuse J, Boku N, et al: Phase II study of gemcitabine chemotherapy alone for locally advanced pancreatic carcinoma: JCOG0506. Jpn J Clin Oncol 40: 573-579, 2010.

3. Evans DB, Abbruzzese JL and Willett CG: Cancer of the pancreas. In: Cancer: Priciples and Practice of Oncology. 6th edition. De Vita, Hellman S and Rosenberg SA (eds). Lippincott, Williams and Wilkins, Philadelphia, pp1126-1161, 2001.

4. Oettle H, Post S, Neuhaus P, et al: Adjuvant chemotherapy with gemcitabine vs. observation in patients undergoing curativeintent resection of pancreatic cancer: a randomized controlled trial. JAMA 297: 267-277, 2007.

5. Aloia TE, Lee JE, Vauthey JN, et al: Delayed recovery after pancreaticoduodenectomy: a major factor impairing the delivery of adjuvant chemotherapy? J Am Coll Surg 204: 347-355, 2007.

6. Sandy H, Bruckner H, Cooperman A, Paradiso J and Kiefer L: Survival advantage of combined chemoradiotherapy compared with resection as the initial treatment of patients with regional pancreatic carcinoma. An outcomes trial. Cancer 89: 314-327, 2000.

7. Ensminger WD, Rosowsky A and Raso V: A clinical pharmacological evaluation of hepatic arterial infusions of 5-fluoro-2-deoxyuridine and 5-fluorourcil. Cancer Res 38: 3784-3792, 1978.

8. Vogl TJ, Schwarz W, Eichler K, et al: Hepatic intraarterial chemotherapy with gemcitabine in patients witn unresectable cholangiocarcinoma and liver metastases of pancreatic cancer: a clinical study on maximum tolerable dose and treatment efficacy. J Cancer Res Clin Oncol 132: 745-755, 2006.

9. Tse AN, Wu N, Patel D, Haviland D and Kemeny N: A phase I study of gemcitabine given via intrahepatic pump for primary or metastatic hepatic malignancies. Cancer Chemother Pharmacol 64: 935-944, 2009.

10. Van Riel JM, Peters GJ, Mammatas LH, et al: A phase I and pharmacokinetic study of gemcitabine given by $24-\mathrm{h}$ hepatic arterial infusion. Eur J Cancer 45: 2519-2527, 2009.

11. Tajima $\mathrm{H}$, Ohta $\mathrm{T}$, Kitagawa $\mathrm{H}$, et al: Hepatic arterial infusion chemotherapy for post-operative liver metastases from pancreatic cancer in a patient with leukocytopenia: a case report. Exp Therap Med 1: 987-990, 2010.

12. Urata K, Kawasaki S, Matsunami T, et al: Calculation of child and adult standard liver volume for liver transplantation. Hepatology 21: 1317-1321, 1995.

13. Therasse P, Arbuck SG, Eisenhauer EA, et al: New guidelines to evaluate the response to treatment in solid tumors. European Organization for Research and Treatment of Cancer, National Cancer Institute of the United States. National Cancer Institute of Canada. J Natl Cancer Inst 92: 205-216, 2000.

14. Nagakawa T, Nagamori M, Futakami F, et al: Result of extensive surgery for pancreatic carcinoma. Cancer 77: 640-645, 1996.

15. Noto M, Miwa K, Kitagawa H, et al: Pancreas head carcinoma. Frequency of invasion to soft tissue adherent to the superior mesenteric artery. Am J Surg Pathol 29: 1056-1061, 2005.

16. Spitz FR, Abbruzzese JL, Lee JE, et al: Preoperative and postoperative chemoradiation strategies in patients treated with pancreaticoduodenectomy for adenocarcinoma of the pancreas. J Clin Oncol 15: 928-937, 1997.

17. Hoffman JP, Lipsitz S, Pisansky T, Weese JL, Solin L and Benson AB: Phase II trial of preoperative radiation therapy and chemotherapy for patients with localized, resectable adenocarcinoma of the pancreas: an Eastern Cooperative Oncology Group Study. J Clin Oncol 16: 317-323, 1998. 
18. Palmer DH, Stocken DD, Hewitt H, et al: A randomized phase 2 trial of neoadjuvant chemotherapy in resectable pancreatic cancer: gemicitabine alone versus gemcitabine combined with cisplatin. Ann Surg Oncol 14: 2088-2096, 2007.

19. Golcher H, Brunner T, Grabenbauer G, et al: Preoperative chemoradiation in adenocarcinoma of the pancreas. A single centre experience advocating a new treatment strategy. Eur J Surg Oncol 34: 756-764, 2008

20. Turrini O, Viret F, Zabotto LM, et al: Neoadjuvant 5 fluorouracil-cisplatin chemoradiation effect on survival in patients with resectable pancreatic head adenocarcinoma: a ten-year single institution experience. Oncology 76: 413-419, 2009.

21. Takai S, Satoi S, Yanagimoto H, et al: Neoadjuvant chemoradiation in patients with potentially resectable pancreatic cancer. Pancreas 36: 26-32, 2008

22. Satoi S, Yanaagimoto H, Toyokawa H, et al: Surgical results after prepoerative chemoradiation therapy for patients with pancreatic cancer. Pancreas 38: 282-288, 2009.

23. Cohen AD and Kemeny NE: An update on hepatic arterial infusion chemotherapy for colorectal cancer. Oncologist 8: 553-566, 2003.

24. Mocellin S, Pilati P, Lise M and Nitti D: Meta-analysis of hepatic arterial infusion for unresectable liver metastases from colorectal cancer: the end of an era? J Clin Oncol 25: 5649-5654, 2007.
25. Homma H, Akiyama T, Mezawa S, et al: Advanced pancreatic carcinoma showing a complete response to arterial infusion chemotherapy. Int J Clin Oncol 9: 197-201, 2004.

26. Miyanishi K, Ishiwatari $\mathrm{H}$ and Hayashi T: A phase I trial of arterial infusion chemotherapy with gemcitabine and 5-fluorouracil for unresectable advanced pancreatic cancer after vascular supply distribution via superselective embolization. Jpn J Clin Oncol 38: 268-274, 2008

27. Mogami K, Ichihara T, Sato T, et al: A case of the papilla of vater accompanied witn a stricture of the celiac artery by the median arcuate ligament. Jpn J Gastroenterol Surg 41: 1588-1593, 2008

28. Kikuchi K and Kanno H: Comparison for blood levels and clinical effects between tablet and other dosage forms of 5-fluorouracil (5-FU). Gan To Kagaku Ryouho 6: 559-565, 1979.

29. Maruyama S, Ando M and Watayo T: Concentration of 5-FU after hepatic artery infusion chemotherapy for liver metastases of colorectal cancer. Gan To Kagaku Ryouho 30: 1635-1638, 2003. 